\title{
HIGH TEMPERATURE SU(2) GLUON MATTER ON THE LATTICE
}

\author{
J. ENGELS, F. KARSCH and H. SATZ \\ Department of Theoretical Physics, University of Bielefeld, Germany \\ and \\ I. MONTVAY \\ II. Institut für Theoretische Physik der Universität Hamburg ${ }^{1}$, Germany
}

Received 21 January 1981

\begin{abstract}
We calculate by Monte Carlo simulation on the lattice the energy density $\mathrm{c}$ of an SU (2) Yang-Mills system at finite physical temperature. First, we study the high temperature form of $\epsilon$, showing that the conventional euclidean lattice formulation converges to the parameter-free Stefan-Boltzmann limit of a free gluon gas in the continuum. Secondly, we show that the specific heat of gluon matter exhibits a sharp peak at the transition poirit from the confined phase to the colorscreened gluon gas. The resulting transition temperature is found to be $210 \pm 10 \mathrm{MeV}$.
\end{abstract}

Recent Monte Carlo studies of SU(2) Yang-Mills systems [1,2] have provided strong indications that gluon matter experiences a phase transition at a critical temperature $T_{\mathrm{c}}$ around $160-230 \mathrm{MeV}$, changing from a confined phase below $T_{\mathrm{c}}$ to one in which color Debye screening renders the gluons effectively free, above $T_{\mathrm{c}}$. Such a transition was expected from strong coupling considerations [3] as well as from a variety of phenomenological approaches [4]; nevertheless, Monte Carlo calculations on the lattice so far constitute the only way of treating in one approach the entire range from confinement to "free" gluons.

The aim of this note is to further investigate by Monte Carlo methods the thermodynamics of finite temperature gluon matter. First, we want to connect at high temperature the lattice formulation with the results of perturbative QCD [5-7] in the continuum, where the Stefan-Boltzmann form of the energy density provides a completely determined parameterfree limit. As second point, we shall show that the specific heart of gluon matter exhibits a singularitylike peak at the deconfinement transition - giving us a very physical and clear-cut way to determine the transition temperature $T_{\mathrm{c}}$.

1 Supported by the Bundesministerium für Forschung und Technologie, Bonn, Germany.
We start, as in refs. $[1,2]$, from the lattice partition function

$Z\left(N, N_{\beta}, g^{2}\right) \equiv \int \prod_{\{i, j\}} \mathrm{d} U_{i j} \exp \left[-\left(4 / g^{2}\right) S(U)\right]$,

where $\mathrm{d} U_{i j}$ is the invariant $\mathrm{SU}(2)$ measure and $U_{i j}$ the corresponding link variable; $g$ denotes the bare coupling constant. The action

$S=\sum_{\{P\}}\left(1-\frac{1}{2} \operatorname{tr} U_{i j} U_{j k} U_{k l} U_{l i}\right)$,

is a sum over all plaquettes $\{P\}$, where $i, j, k, l$ label the sites defining the plaquette. The lattice underlying eqs. (1) and (2) has $N$ sites in each spatial direction and $N_{\beta}$ sites in the temperature (= imaginary time) direction.

Eq. (2) holds for equal lattice spacing in all directions. Choosing the lattice spacing in the temperature direction, $a_{\beta}$, to be different from the lattice spacing in the spatial directions, $a$, eq. (2) goes over into

$$
\begin{aligned}
S= & \left(\frac{a_{\beta}}{a} \sum_{\left\{P_{\mathrm{s}}\right\}}\left(1-\frac{1}{2} \operatorname{tr} U_{i j} \ldots U_{l i}\right)\right. \\
& \left.+\frac{a}{a_{\beta}} \sum_{\left\{P_{\beta}\right\}}\left(1-\frac{1}{2} \operatorname{tr} U_{i j} \ldots U_{l i}\right)\right) .
\end{aligned}
$$


Here the "space-like" plaquettes $\left\{\boldsymbol{P}_{\mathbf{s}}\right\}$ have only links in spatial directions and the "temperature-like" plaquettes $\left\{P_{\beta}\right\}$ have two (opposite) links in the temperature direction.

Imposing periodic boundary conditions in the temperature direction, for sufficiently large $N$ and $N_{\beta}$ but fixed $\beta=N_{\beta} a_{\beta}$, this lattice describes a system of temperature $T=\beta^{-1}$. Periodic boundary conditions in the spatial directions (although not necessary) are usually also assumed. In the thermodynamic limit the overall spatial volume $V=(\mathrm{Na})^{3}$ has to be large enough for the spatial surface effects to become negligible. Therefore, generally speaking, in Monte Carlo simulations of finite temperature systems, $N_{\beta} \ll N$ is required (at least for $a_{\beta}=a$ ) in order to minimize the finite volume effects in comparison with the effects of periodicity in the temperature direction. The temperature $T=0$ is obtained by holding $a$ and $a_{\beta}$ fixed as $N$ and $N_{\beta}$ become "infinite". Note that there is no particular connection between $T=0$ and a finite symmetric lattice, just as there is no reason, other than simplicity, for spatially symmetric lattices. $N_{\mu}$ must just be large enough in each direction $\mu$ to leave the results insensitive to a change of $N_{\mu}$. (For a discussion of finite temperature effects in previous Monte Carlo lattice calculations see ref. [8].)

In the continuum, the energy density $\epsilon$ is given by

$\epsilon=-V^{-1}[\partial \ln Z(\beta, V) / \partial \beta]_{V}$,

and the pressure $p$ by

$p=\beta^{-1}[\partial \ln Z(\beta, V) / \partial V]_{\beta}$.

On the lattice, with fixed $N$ and $N_{\beta}$, we write

$\partial / \partial \beta=N_{\beta}^{-1} \partial / \partial a_{\beta}$,

$\partial / \partial V=\left(3 N^{3} a^{2}\right)^{-1} \partial / \partial a$,

for the derivatives in eqs. (4) and (5). With eqs. (1) and (3), this yields

$\epsilon=\epsilon_{a}+\epsilon_{g}, \quad p=p_{a}+p_{g}$

for energy density and pressure, where

$$
\begin{aligned}
\epsilon_{a} & \equiv 4\left(N^{3} N_{\beta} a^{3} a_{\beta} g^{2}\right)^{-1}\left\langle\frac{a_{\beta}}{a} \sum_{\left\{P_{\mathrm{s}}\right\}}\left[1-\frac{1}{2} \operatorname{tr} U_{i j} \ldots U_{l i}\right]\right. \\
& \left.-\frac{a}{a_{\beta}} \sum_{\left\{P_{\beta}\right\}}\left[1-\frac{1}{2} \operatorname{tr} U_{i j} \ldots U_{l i}\right]\right\rangle
\end{aligned}
$$

and, with eq. (3),

$\epsilon_{g} \equiv 4\left(N^{3} N_{\beta} a^{3}\right)^{-1}\left(\partial g^{-2} / \partial a_{\beta}\right)_{a}\langle S\rangle$,

$p_{g} \equiv-\frac{4}{3}\left(N^{3} N_{\beta} a^{2} a_{\beta}\right)^{-1}\left(\partial g^{-2} / \partial a\right)_{a_{\beta}}\langle S\rangle$.

Here \langle\rangle denotes, as usual, the thermodynamic average over the partition function (1).

The pressure $p_{a}$ and energy density $\epsilon_{a}$ "at constant $g$ " are found to satisfy the zero mass relation

$p_{a}=\frac{1}{3} \epsilon_{a}$,

already on the lattice.

In the continuum, perturbation theory gives as high temperature limit [5-7]

$$
\begin{aligned}
\epsilon= & \left(N_{g} \pi^{2} / 15\right) T^{4}\left[1-\alpha_{\mathrm{s}} 5 N_{\mathrm{c}} / \pi\right. \\
& \left.+\alpha_{\mathrm{s}}^{3 / 2} 80\left(N_{\mathrm{c}} / \pi\right)^{3 / 2} / \sqrt{3} \pm \ldots\right],
\end{aligned}
$$

where $N_{\mathrm{g}}=N_{\mathrm{c}}^{2}-1$ denotes the number of gluons [3 for $\mathrm{SU}(2)]$ and $N_{\mathrm{c}}$ the number of colors [2 for SU(2)] ; $\alpha_{\mathrm{s}}$ is the running coupling constant of QCD

$\alpha_{\mathrm{s}}=g_{\mathrm{s}}^{2} / 16 \pi=3 \pi / 22 N_{\mathrm{c}} \ln (4 T / \Lambda)$,

with $\Lambda$ as the continuum normalization parameter. For sufficiently high temperatures, the continuum limit of the SU(2) lattice system must thus attain the StefanBoltzmann form

$\epsilon_{\mathrm{SB}}=\frac{1}{5} \pi^{2} T^{4}$,

independently of the choice of continuum or lattice normalization parameters $\Lambda$ and $\Lambda_{L}$. One of our aims is to check if eq. (8) indeed converges to this parameter-free limit. In view of the complex relationship between euclidean lattice and hamiltonian continuum formulations, such a convergence is not a priori evident.

The lattice spacing $a$, the lattice scale parameter $\Lambda_{\mathrm{L}}$ and the coupling $g$ are in the connected limit $(a \rightarrow 0)$ connected by the renormalization group relation

$\Lambda_{\mathrm{L}} a=\left(11 g^{2} / 24 \pi^{2}\right)^{-51 / 121} \exp \left(-12 \pi^{2} / 11 g^{2}\right)$,

Monte Carlo calculations have yielded [9]

$\Lambda_{\mathrm{L}}=(1.3 \pm 0.2) \times 10^{-2}[\sigma(0)]^{1 / 2}$

for $\Lambda_{\mathrm{L}}$ in terms of the string tension $\sigma(0)$ at $T=0$. With the relation $\sigma(0)=\left(2 \pi \alpha^{\prime}\right)^{-1}$ and a Regge slope $\alpha^{\prime}=1$ $(\mathrm{GeV})^{-2}$, this gives

$\Lambda_{\mathrm{L}}=5.2 \pm 0.8 \mathrm{MeV}$. 


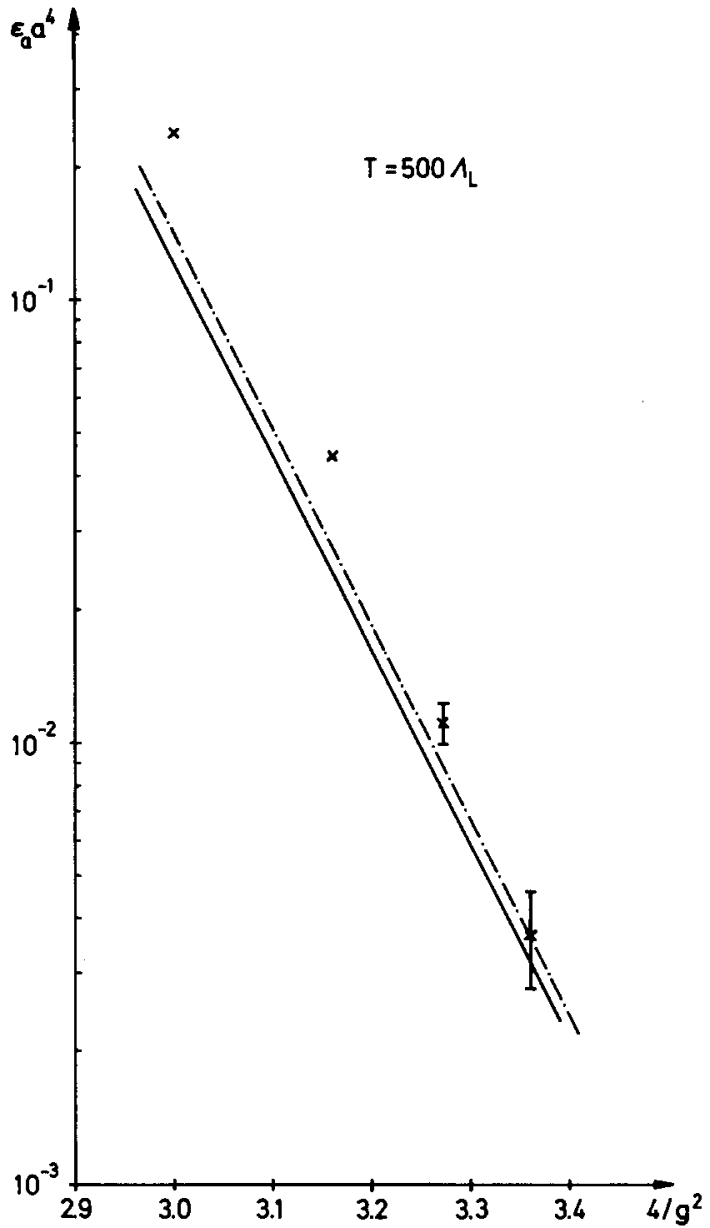

Fig. 1. Energy density of gluon matter versus $4 / g^{2}$, at fixed temperature $T=500 \Lambda_{\mathrm{L}}$, after about 600 iterations. The solid line gives the Stefan-Boltzmann limit; the dashed line includes the perturbative corrections of eq. (12).

This value of $\Lambda_{L}$, when inserted in the SU(2) relation [10]

$\Lambda \approx 57.5 \Lambda_{\mathrm{L}}$

leads to $\Lambda \approx 300 \pm 50 \mathrm{MeV}$, in reasonable consistency with what one would expect from deep inelastic scattering or $\mathrm{e}^{+} \mathrm{e}^{-}$annihilation data. - These connections being given, we shall from now on, for convenience, measure $T$ in units of $\Lambda_{\mathrm{L}}$.

To calculate the "constant $g$ " part (9) of the lattice energy density, we consider a $10^{3} \times N_{\beta}$ lattice with isotropic spacing $a=a_{\beta}$. Holding the temperature $T$ $=\left(N_{\beta} a\right)^{-1}$ fixed, we let $N_{\beta}$ run from $N_{\beta}=2$ to $N_{\beta}=5$

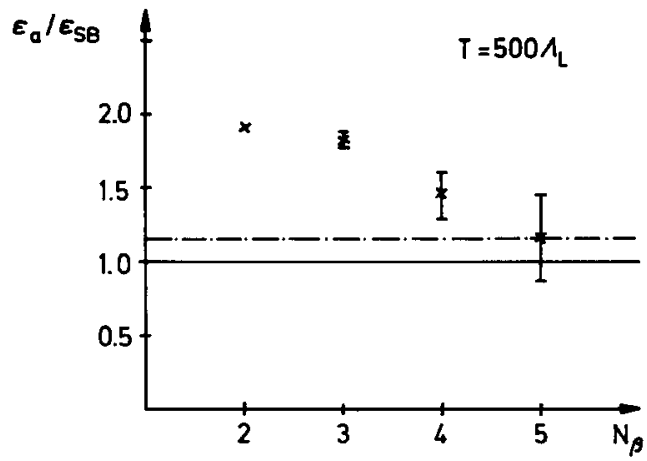

Fig. 2. Energy density of gluon matter versus lattice size, at $T=500 \Lambda_{\mathrm{L}}$, in comparison with the Stefan-Boltzmann limit ${ }_{\mathrm{ESB}}$; the dashed line lincludes the perturbative corrections of eq. (12).

(varying $a$ with $N_{\beta}$ accordingly). We can now ask two questions: Has the energy density $\epsilon_{a}$ at the spacing $a$ corresponding to a given $T$ already reached the continuum limit? If this is the case, then the relation between $g$ and $a$ is given by eq. (15). Does $\epsilon_{a}$ at high temperature approach the Stefan-Boltzmann form? This need not be the case, since we have so far ignored the second term in eq. (8).

If $\epsilon_{a}$ does satisfy the Stefan-Boltzmann relation

$\epsilon_{a} a^{4}=\pi^{2} /\left(5 N_{\beta}^{4}\right)$,

at a given temperature $T=\tau \Lambda_{\mathrm{L}}$, then we have

$\epsilon_{a} a^{4}=\frac{1}{5} \pi^{2} \tau^{4}$

$$
\times\left[\left(11 g^{2} / 24 \pi^{2}\right)^{-51 / 121} \exp \left(-12 \pi^{2} / 11 g^{2}\right)\right]^{4}
$$

if at the lattice spacing $a=\left(N_{\beta} \tau \Lambda_{\mathrm{L}}\right)^{-1}$, which corresponds to $g$, the energy density $\epsilon_{a}$ is in the continuum limit. Relation (20) thus gives us a parameter-free $g$ $\rightarrow 0$ and high temperature limit.

In fig. 1 we show for $\tau=500$ (corresponding to $T$ $\approx 2.6 \mathrm{GeV}$ ) the result of our Monte Carlo calculations, using the same methods as ref. [9]. Besides the Stefan-Boltzmann limit (20), we also display the limiting curve obtained by including the higher order corrections of eq. (12), using $\Lambda / \Lambda_{\mathrm{L}}=57.5$. In spite of the relatively small lattice size (manageable for computers), both the absolute value and the $g$-dependence of our Monte Carlo results are seen to agree quite well with the free gluon gas limit. To illustrate the dependence of our results on the lattice size, we shown in fig. 2 a 


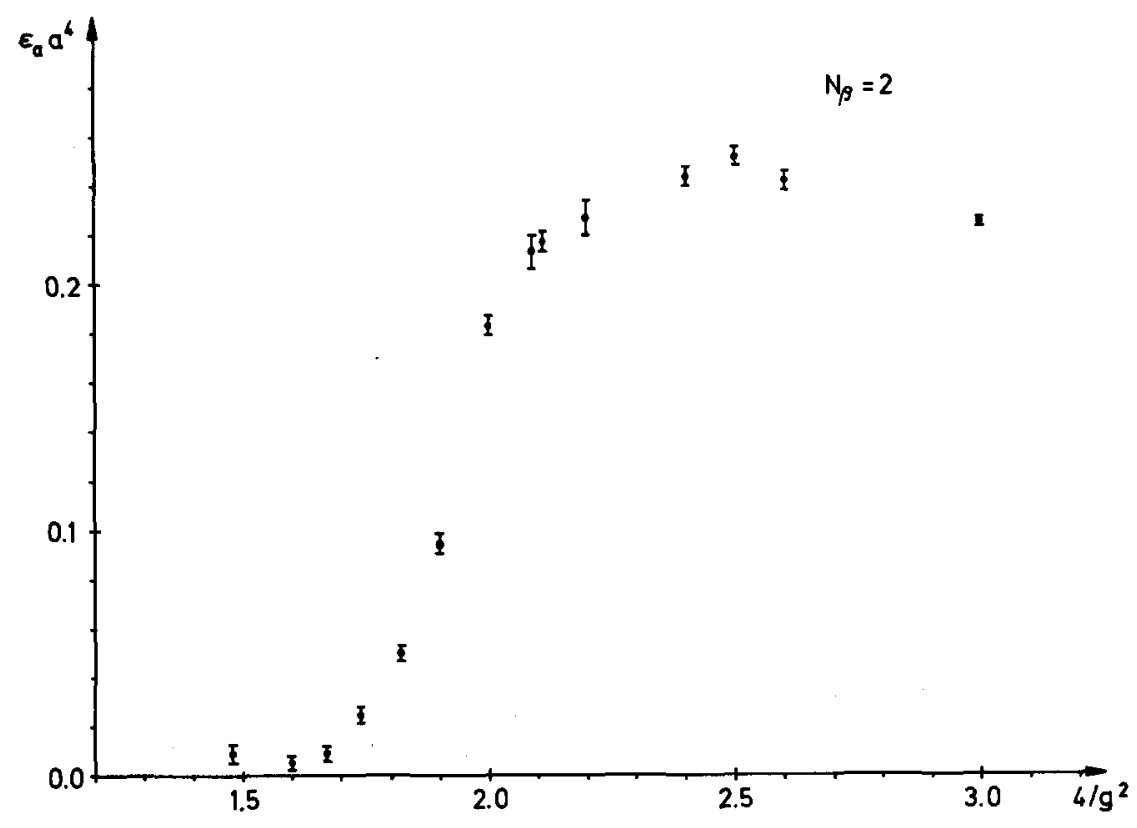

Fig. 3. Energy density of gluon matter versus $4 / g^{2}$, at fixed lattice size $N_{\beta}=2$, after about 500 iterations.

plot of $\epsilon_{a} / \epsilon_{\mathrm{SB}}$ as function of $N_{\beta}$; the convergence to the free gluon gas appears to be fairly rapid.

So far, we have considered only the first term $\epsilon_{a}$ of eq. (8), and that alone was seen to yield the correct Stefan-Boltzmann limit. To check that the second term, $\langle S\rangle\left(\partial g^{-2} / \partial a_{\beta}\right)_{a}$, is indeed negligible, we have numerically calculated the variation of $g$ as a function of $a_{\beta}$ at fixed $a$.

The best way to define the lattice coupling constant $g$ in a finite temperature calculation is to choose a dimensional thermodynamic quantity and fix its physical value in terms of $g$. (This is analogous to the usual procedure of fixing in field theory at $T=0$, say, the physical value of the string tension in order to define the dependence of $g$ on $a[9,11]$.) An obvious choice in the gluon system is to fix the physical value of the critical temperature for the deconfining phase transition. It is convenient to introduce, instead of $a$ and $a_{\beta}$, the variables $t_{\mathrm{c}}$ and $\alpha$ :

$t_{\mathrm{c}} \equiv a T_{\mathrm{c}}, \quad \alpha \equiv a_{\beta} / a$

implying

$$
\begin{aligned}
& a\left(\partial g^{-2} / \partial a\right)_{a_{\beta}}=t_{\mathrm{c}}\left(\partial g^{-2} / \partial t_{\mathrm{c}}\right)_{\alpha}-\alpha\left(\partial g^{-2} / \partial \alpha\right)_{t_{\mathrm{c}}} \\
& a\left(\partial g^{-2} / \partial a_{\beta}\right)_{a}=\left(\partial g^{-2} / \partial \alpha\right)_{t_{\mathrm{c}}} .
\end{aligned}
$$

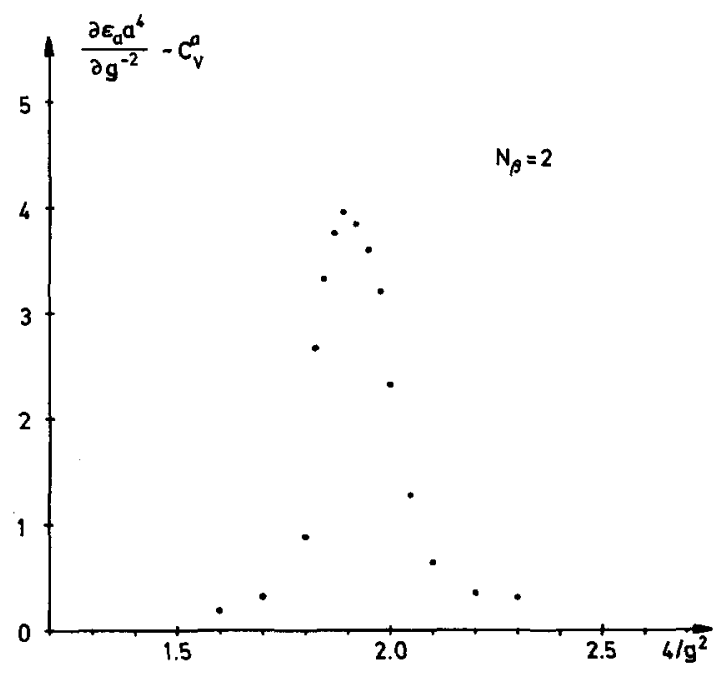

Fig. 4. Specific heat of gluon matter versus $4 / g^{2}$, at fixed lattice size $N_{\beta}=2$.

From the connection of the lattice spacing $a$ with the lattice scale parameter $\Lambda_{L}$ in eq. (15), there follows for small $g$, independently of the value of $\Lambda_{L}$ :

$$
t_{\mathrm{c}}\left(\partial \mathrm{g}^{-2} / \partial t_{\mathrm{c}}\right)_{\alpha=1} \rightarrow-11 /\left(12 \pi^{2}\right) \text {. }
$$

The value of $\left(\partial g^{-2} / \partial \alpha\right)_{t_{\mathrm{c}}}$ can be determined numeri- 
cally by looking for the variation of the critical value of $g^{-2}$ which corresponds to $T_{\mathrm{c}}$. When we go, for instance, from $N_{\beta}=4$ and $\alpha=1$ to $N_{\beta}=3$ and $\alpha$ $=4 / 3$ the value of $a$ and $t_{\mathrm{c}}$ is unchanged. The change in the critical value of $g^{-2}$ gives a numerical estimate for $\left(\partial g^{-2} / \partial \alpha\right)_{t}$. The resulting value in our range of $g^{2}$ (about $g^{2}=1-2$ ) is small (of the order of a few percent of $g^{-2}$ ).

In $\epsilon_{g}$, the derivative $\left(\partial g^{-2} / \partial \alpha\right)_{t}$ is multiplied by the average action $\langle S\rangle$, which is not small and does not vanish exponentially for $g \rightarrow 0$, as would be required by the renormalization group. However, $\epsilon$ still contains the zero point term ("vacuum energy density") inherent in the euclidean formulation [12].

This term is infinite for $g \rightarrow 0$, but it does not depend on the temperature. Hence it can be removed by calculating the differences of $\langle S\rangle$ between two temperatures at fixed $g$. These differences, multiplied by $\left(\partial g^{-2} / \partial \alpha\right)_{t_{c}}$, are less than $1 \%$ of $\epsilon_{a}$ in the temperature range we checked $\left(T \gtrsim 100 \Lambda_{\mathrm{L}}\right)$. Hence $\epsilon_{a}$ is for $a=a_{\beta}$ indeed a good approximation of the energy density at these temperatures.

Having established the connection between the lattice energy density and its high temperature continuum limit, we now turn to the temperature region around the deconfinement transition. In fig. 3, we show the behaviour of $\epsilon_{a}$ for $N_{\beta}=2$ as function of $g^{2}$. Apart from a scale change, this also gives us the temperature dependence of $\epsilon_{a}$, since at fixed $N_{\beta}$ the coupling $g$ and the lattice spacing $a$ are related either by the renormalization group relation (15), or, in the nonasymptotic regime, by numerical [9] and/or strong coupling $[11,13]$ results. The corresponding specific heat

$C_{V}^{a}=\partial \epsilon_{a} / \partial T \approx \partial \epsilon_{a} a^{4} / \partial g^{-2}$,

is shown in fig. 4 . At $4 / g^{2} \approx 1.90$ (for $N_{\beta}=2$ ) we have a clear peak as the signal of the deconfinement transition from gluonium matter to gluon gas. (Due to the sharpness of the peak, its position is not changed noticeably if we differentiate with respect to $g^{-2}$ instead of T.)

To show that the transition observed here in terms of the specific heat is indeed the deconfinement transition defined in refs. [1,2] through the average Wilson loop $|\langle L\rangle|$, we display in fig. 5 our results for $|\langle L\rangle|$ at $N_{\beta}=2$. The change from $\langle L\rangle \approx 0$ in the confined phase to $\langle L\rangle \neq 0$ in the gluon gas is seen to occur at the

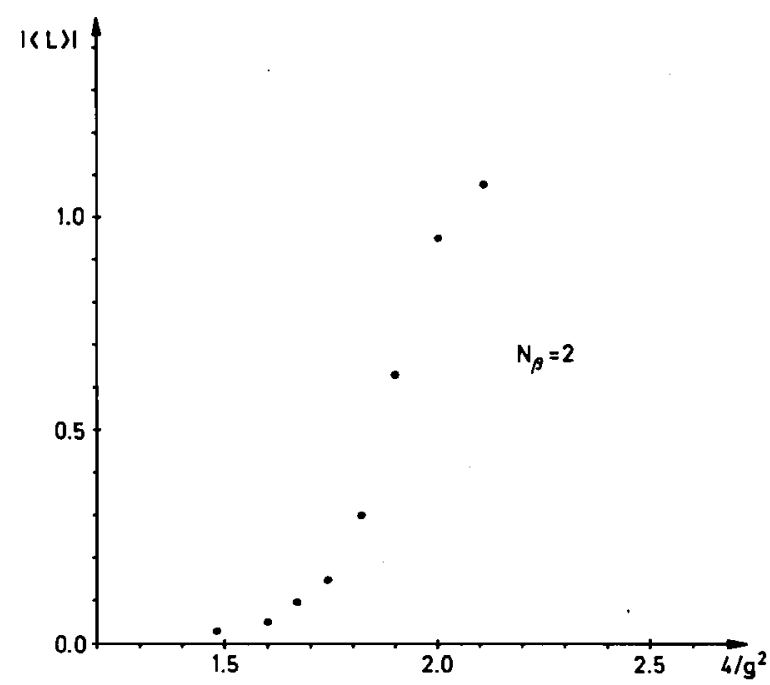

Fig. 5. Average Wilson loop versus $4 / g^{2}$, at fixed lattice size $N_{\beta}=2$.

same $g^{2}$ as the rise of $\epsilon_{a}$ in fig. 3. We note that a determination of $T_{\mathrm{c}}$ through the peak of the specific heat, which seems physicallly the most clear-cut, leads to a higher $T_{\mathrm{c}}$ than what one obtains by placing the transition at that $g^{2}$ where $\langle L\rangle$ becomes non-zero. This may account for the lower $T_{\mathrm{c}}$ found in ref. [1]

To assure that the transition occurs indeed at fixed temperature, we have also calculated $C_{V}^{a}$ for $N_{\beta}=4$. The peak then lies at $4 / g^{2} \approx 2.28$; using eq. (15), this gives $T_{\mathrm{c}}=40 \pm 2 \Lambda_{\mathrm{L}}$, which by eq. (17) yields $T_{\mathrm{c}}=210$ $\pm 10 \mathrm{MeV}$. This is in agreement with the value obtained for $N_{\beta}=2$, if there the non-asymptotic part of the curve of Creutz [9] is used.

Finally we note that at lower temperatures, in the region of $T_{\mathrm{c}}$, both energy density and specific heat will in addition have contributions from $\epsilon_{g}$; these, however, are not expected to modify significantly the location of the transition.

\section{References}

[1] J. Kuti, J. Polónyi and K. Szlachányi, Phys. Lett. 98B (1981) 199.

[2] L.D. McLerran and B. Svetitsky, Phys. Lett. 98B (1981) 195.

[3] L. Susskind, Phys. Rev. D20 (1979) 2610.

[4] For a review, see e.g. H. Satz, On critical phenomena in strong interaction physics, Bielefeld preprint BI-TP 80/33 (1980); to be published in: Proc. 17th Winter School of Theoretical physica (Karpacz, Poland). 
[5] J.I. Kapusta, Nucl. Phys. B148 (1979) 461.

[6] O.K. Kalashnikov and V.V. Klimov, Phys. Lett. 88B (1979) 328.

[7] For a review, see e.g.: M. Kislinger and P. Morley, Phys. Rep. 51 (1979) 64.

[8] J. Engels, F. Karsch, I. Montvay and H. Satz, Finite lattice effects in Monte Carlo simulations of SU(2) gluon systems, to be published.
[9] M. Creutz, Phys. Rev. D21 (1980) 2308.

[10] A. Hasenfratz and P. Hasenfratz, Phys. Lett. 93B (1980) 165.

[11] J.B. Kogut, R.B. Pearson and J. Shigemitsu, Phys. Rev. Lett. 43 (1979) 484.

[12] C. Bernard, Phys. Rev. D9 (1974) 3312.

[13] G. Münster, Phys. Lett. 95B (1980) 59. 